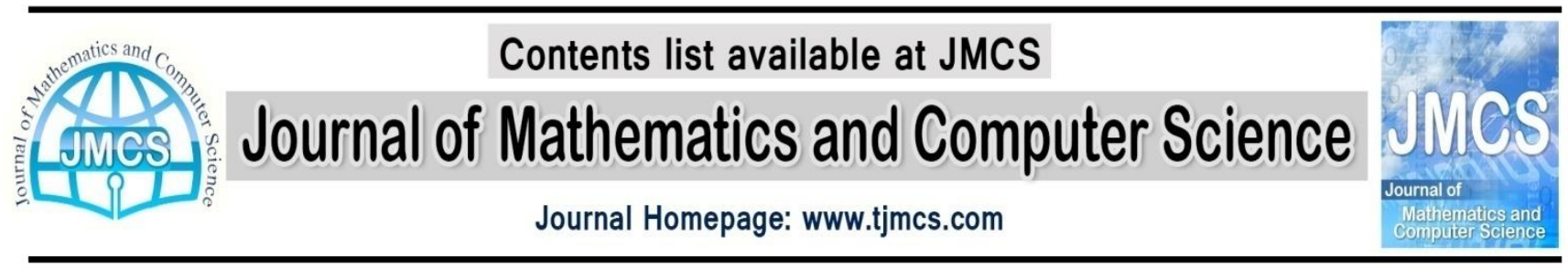

\title{
Generalized Normed Spaces and Fixed Point Theorems
}

\author{
Kamran Alam Khan \\ Department of Mathematics, V. R. A. L. Govt. Girls P. G. College, Bareilly (U.P.)-INDIA \\ Email: kamran12341@yahoo.com
}

\begin{abstract}
Article history:
Received July 2014

Accepted September 2014

Available online October 2014
\end{abstract}

\begin{abstract}
Gähler ([4], [5]) introduced and investigated the notion of 2-metric spaces and 2-normed spaces in sixties. These concepts are inspired by the notion of area in two dimensional Euclidean space. In this paper, we choose a fundamentally different approach and introduce a possible generalization of usual norm retaining the distance analogue properties. This generalized norm will be called as $G$-norm. We show that every G-normed space is a G-metric space and therefore, a topological space and develop the theory for G-normed spaces. We also introduce G-Banach spaces and obtain some fixed point theorems.
\end{abstract}

Keywords: Linear 2-normed space, 2-metric space, G-normed space, G-metric space, Fixed point theorem

2010 Mathematics Subject Classification: Primary 46B20, 47H10; Secondary 46B07, 47A30

\section{Introduction}

Gähler ([4], [5]) introduced and studied the concept of 2-metric spaces and 2-normed spaces and extended the theory to n-normed spaces in ([6], [7], [8]). Since then many authors ([2], [3], [9], [10], [12], [6] etc.) have published a number of articles devoted to these concepts. It was mentioned by Gähler [4] that the notion of a 2-metric is an extension of an idea of ordinary metric. The usual metric is a kind of generalization of the notion of distance whereas the concept of a 2-metric and hence that of a 2-norm are inspired by the notion of area in two dimensional Euclidean space and geometrically $d(x, y, z)=0$ represents the area of a triangle formed by the points $\mathrm{x}, \mathrm{y}$ and $\mathrm{z}$ in $X$ as its vertices. But this is not always true. Sharma [15] showed that $d(x, y, z)=0$ for any three distinct points $x, y, z \in \mathbb{R}^{2}$. Also K. S. Ha et al [10] have shown that in many cases there is no connection between the results obtained in the usual metric spaces and 2-metric spaces. 
B. C. Dhage [1] attempted to generalize the concept of usual metric and introduced the concept of Dmetric in order to translate results from usual metric space to D-metric space. But the topological structure of D-metric spaces was found to be incorrect ([13]). Finally, Mustafa and Sims ([14]) introduced the concept of G-metric in which the tetrahedral inequality is replaced by an inequality involving repetition of indices. This new approach is fundamentally different from that of Gähler and retains the notion of distance. Recently the author ([11]) generalized the concept to $n(\geq 3)$ variables and introduced Generalized n-metric spaces. In this paper our aim is to generalize the concept of normed space in such a manner that the generalized norm retains the distance analogue properties of the usual norm. We call this generalized norm a G-norm. We show that every G-normed space is a G-metric space and therefore, a topological space. Hence the topological concepts such as open subset, closed subset, limit, closure etc make sense. We develop the theory for G-normed spaces and also introduce G-Banach spaces. Finally we obtain some fixed point theorems. Let us begin with some definitions-

Definition 1.1 ([5]) Let $X$ be a real linear space of dimension greater than one and let $\|.,$.$\| be a real$ valued function on $X \times X$ satisfying the following conditions:

(1) $\|x, y\| \geq 0$ for every $x, y \in X$; $\|x, y\|=0$ if and only if $x$ and $y$ are linearly dependent,

(2) $\|x, y\|=\|x, y\|$ for every $x, y \in X$,

(3) $\|\alpha x, y\|=|\alpha|\|x, y\|$ for every $x, y \in X$ and $\alpha \in \mathbb{R}$,

(4) $\|x, y+z\| \leq\|x, y\|+\|x, z\|$ for every $x, y, z \in X$.

Then the function $\|.,$.$\| is called a 2-norm on X$ and the pair $(X,\|.,\|$.$) a linear 2-normed space.$

Example 1.1.1 Let $X=\mathbb{R}^{3}$ and $x, y \in \mathbb{R}^{3}$ such that $x=\left(x_{1}, x_{2}, x_{3}\right)$ and $y=\left(y_{1}, y_{2}, y_{3}\right)$. Define

$$
\|x, y\|=\max \left\{\left|x_{1} y_{2}-x_{2} y_{1}\right|+\left|x_{1} y_{3}-x_{3} y_{1}\right|,\left|x_{1} y_{2}-x_{2} y_{1}\right|+\left|x_{2} y_{3}-x_{3} y_{2}\right|\right\}
$$

Then $\left(\mathbb{R}^{3},\|.,\|.\right)$ is a 2-normed space.

Definition 1.2 ([14]) Let $X$ be a non-empty set, and $\mathbb{R}^{+}$denote the set of non-negative real numbers. Let $G: X \times X \times X \rightarrow \mathbb{R}^{+}$be a function satisfying:

[G 1] $G(x, y, z)=0$ if $x=y=z$,

[G 2] $G(x, x, y)>0 x, y \in X$ with $x \neq y$,

[G 3] $G(x, x, y) \leq G(x, y, z)$ for all $x, y, z \in X$,

[G 4] $G(x, y, z)=G(y, z, x)=G(x, z, y)=\cdots$ for all $x, y, z \in X$,

[G 5] $G(x, y, z) \leq G(x, a, a)+G(a, y, z)$ for all $x, y, z, a \in X$

Then the function $\mathrm{G}$ is called a generalized metric, or more specifically a G-metric on $X$, and the pair $(X, G)$ a G-metric space.

Example 1.2.1 Let $\mathbb{R}$ denote the set of all real numbers. Define a function $\rho: \mathbb{R} \times \mathbb{R} \times \mathbb{R} \rightarrow \mathbb{R}^{+}$by

$$
\rho(x, y, z)=\max \{|x-y|,|y-z|,|z-x|\} \text { for all } x, y, z \in \mathbb{R} .
$$

Then $(\mathbb{R}, \rho)$ is a $\mathrm{G}$-metric space. 


\section{Main Results}

Definition 2.1 Let $X$ be a real vector space. A real valued function $\|., \ldots\|:, X^{3} \rightarrow \mathbb{R}$ is called a $G$-norm on $X$ if the following conditions hold:

[N 1] $\|x, y, z\| \geq 0$ and $\|x, y, z\|=0$ if and only if $x=y=z=0$,

[N 2] $\|x, y, z\|$ is invariant under permutations of $x, y$ and $z$,

[N 3] $\|\alpha x, \alpha y, \alpha z\|=|\alpha|\|x, y, z\|$ for all $\alpha \in \mathbb{R}$ and $x, y, z \in X$,

[N 4] $\left\|x+x^{\prime}, y+y^{\prime}, z+z^{\prime}\right\| \leq\|x, y, z\|+\left\|x^{\prime}, y^{\prime}, z^{\prime}\right\|$ for all $x, y, z, x^{\prime}, y^{\prime}, z^{\prime} \in X$,

[N 5] $\|x, y, z\| \geq\|x+y, 0, z\|$ for all $x, y, z \in X$

The pair $(X,\|., .,\|$.$) is then called a G$-normed space.

Example 2.1.1 In the linear space $X=C[0,1]$ of real valued continuous functions on $[0,1]$ define a function $\|., . .\|:. X^{3} \rightarrow \mathbb{R}$ by

$$
\|f, g, h\|=\max _{0 \leq t \leq 1}\{|f(t)|+|g(t)|+|h(t)|\} \quad(f, g, h \in \mathrm{C}[0,1])
$$

Then $(X,\|., .,\|$.$) is a G$-normed space.

Remark. From [N 4], we see that $\left\|x+x^{\prime}, 0,0\right\| \leq\|x, 0,0\|+\left\|x^{\prime}, 0,0\right\|$. This is similar to triangle inequality in usual normed spaces.

Proposition 2.1 Let $(X,\|., .,\|$.$) be a G$-normed space. Then for all $x, y, z, u, v, w \in X$, we have

$$
|\|x, y, z\|-\|u, v, w\|| \leq\|x-u, y-v, z-w\|
$$

Proof. The result follows directly from the definition of $G$-normed space.

Proposition 2.2 Let $(X,\|., \ldots\|$,$) be a G$-normed space. Then the function $G: X^{3} \rightarrow \mathbb{R}^{+}$defined by

$$
G(x, y, z)=\|x-y, y-z, z-x\|
$$

is a G-metric defined on $X$.

Proof. We see that [G 1] follows from [N 1]. Also $G(x, x, y)=,\|0, y-x, x-y\|>0$ for $x \neq y$. From [N 2] and [N 5] we have

$$
\|x-y, y-z, z-x\|=\|y-z, z-x, x-y\| \geq\|y-x, 0, x-y\|=\|0, x-y, y-x\|
$$

Which gives $G(x, y, z) \geq G(x, x, y)$. Now [G 4] follows from [N 2] and [N 3]. Finally [G 5] holds as we see that for all $x, y, z, a \in X$, we have

$$
\begin{array}{r}
G(x, y, z)=\|x-a+a-y, 0+y-z, a-x+z-a\| \\
\leq\|x-a, 0, a-x\|+\|a-y, y-z, z-a\|
\end{array}
$$




$$
=G(x, a, a)+G(a, y, z) .
$$

Hence the function $G$ thus defined is a $G$-metric and $(X, G)$ is a $G$-metric space.

Thus every $G$-normed space $(X,\|., \ldots\|$,$) will be considered to be a G$-metric space. We have a well defined topology for a $G$-metric space. For $x_{0} \in X, r>0$, the $G$-ball is defined as the set $B_{G}\left(x_{0}, r\right)=$ $\left\{y \in X: G\left(x_{0}, y, y\right)<r\right\}$. The family of all $G$-balls $\left\{B_{G}(x, r): x \in X, r>0\right\}$ is a base of topology $\tau(G)$ on $X$, called the G-metric topology. This G-metric topology coincides with the metric topology corresponding to the metric $d_{G}$ given by $d_{G}(x, y)=G(x, y, y)+G(x, x, y)$ ([14]). Thus every G-metric space and hence every G-normed space is topologically equivalent to a metric space. Now we can transport concepts such as open balls, open subsets, closed subsets, closure etc from metric spaces into the G-normed spaces.

Definition 2.2 Let $(X,\|., .\|$,$) be a G-normed space. For given x_{0} \in X, e \in X$ and $r>0$, we define open ball $B_{e}\left(x_{0}, r\right)$ to be a subset of $X$ given by

$$
B_{e}\left(x_{0}, r\right)=\left\{y \in X:\left\|x_{0}-y, y-e, e-x_{0}\right\|<r\right\}
$$

and the closed ball $B_{e}\left[x_{0}, r\right]$ in $X$ as

$$
B_{e}\left[x_{0}, r\right]=\left\{y \in X:\left\|x_{0}-y, y-e, e-x_{0}\right\| \leq r\right\}
$$

Substituting $y=r u+x_{0} \quad$ in (2.3), we have

$$
B_{e}\left(x_{0}, r\right)=x_{0}+r\left\{u \in X:\left\|u, e^{\prime}-u, e^{\prime}\right\|<1\right\}
$$

where $e^{\prime}=\left(e-x_{0}\right) r^{-1}$. Hence for $x_{0}=0$, we have

$$
B_{e}(0, r)=r\left\{u \in X:\left\|u, e^{\prime}-u, e^{\prime}\right\|<1\right\}=r B_{e^{\prime}}(0,1)
$$

Example 2.2.1 Let $(X,\|, \ldots\|$,$) be a G-normed space such that$

$$
\|x, y, z\|=\|x\|+\|y\|+\|z\|
$$

for all $x, y, z \in \mathbb{R}^{2}$. Where $\|x\|=\left\|\left(x_{1}, x_{2}\right)\right\|=\sqrt{x_{1}^{2}+x_{2}^{2}}$. Then the open ball $B_{e}\left(x_{0}, r\right)$ in $\mathbb{R}^{2}$ will be an open elliptic disc given by

$$
B_{e}\left(x_{0}, r\right)=\left\{y \in \mathbb{R}^{2}:\left\|x_{0}-y\right\|+\|y-e\|<k\right\}
$$

Where $k=r-\left\|e-x_{0}\right\|$.

Suppose hereafter that $X$ is a G-normed space. Now we introduce some definitions and propositions for further theory.

Definition 2.3 A subset $G \subseteq X$ is open in $X$ if for each $x \in G$, there exist $e \in X$ and $r>0$ such that $B_{e}\left(x_{0}, r\right) \subseteq G$.

Definition 2.4 A set $D$ in a G-normed space $X$ is said to be dense in $X$ when it intersects every open set.

Definition 2.5 A sequence $\left\langle x_{n}\right\rangle$ in $X$ is said to be convergent if there exists an element $x \in X$ such that for given $\varepsilon>0$, there exists a positive integer $N$ such that 


$$
l, m, n \geq N \Rightarrow\left\|x_{l}-x, x_{m}-x, x_{n}-x\right\|<\varepsilon
$$

Or equivalently $n \geq N \Rightarrow\left\|x_{n}-x, x_{n}-x, x_{n}-x\right\|<\varepsilon$.

Definition 2.6 A sequence $\left\langle x_{n}\right\rangle$ in $X$ is said to be a Cauchy sequence if given $\varepsilon>0$, there exists a positive integer $N$ such that

$$
l, m, n \geq N \Rightarrow\left\|x_{l}-x_{m}, x_{m}-x_{n}, x_{n}-x_{l}\right\|<\varepsilon
$$

Definition 2.7 A G-normed space is said to be complete if each Cauchy sequence in $X$ converges in $X$.

Definition 2.8 A complete G-normed space is called a G-Banach space.

Definition 2.9 The closure of a subset $E \subseteq X$, denoted by $\bar{E}$, is the set of all $x \in X$ such that there exists a sequence $\left\langle x_{n}\right\rangle$ in $E$ converging to $x$. We say that $E$ is closed if $\bar{E}=E$.

Definition 2.10 A subset $C$ of $X$ is called convex (resp. absolutely convex) if $\alpha C+\beta C \subseteq C$ for every $\alpha, \beta>0($ resp. $\alpha, \beta \in \mathbb{R})$ with $\alpha+\beta=1(\operatorname{resp} .|\alpha|+|\beta| \leq 1)$.

Proposition 2.3 Every convergent sequence in a G-normed space $X$ has a unique limit.

Proof. The proof is straightforward.

Proposition 2.4 Every convergent sequence in a G-normed space $X$ is a Cauchy sequence.

Proof. The result follows directly from the definitions.

Proposition 2.5 The ball $B_{e}\left(x_{0}, r\right)$ is open in $X$.

Proof. Let $z \in B_{e}\left(x_{0}, r\right)$. Then $\left\|x_{0}-e, e-z, z-x_{0}\right\|<r$

Now using [N 5], we get $r>\left\|x_{0}-e, e-z, z-x_{0}\right\| \geq\left\|x_{0}-z, 0, z-x_{0}\right\|$

Let $r_{1}=r-\left\|x_{0}-z, 0, z-x_{0}\right\|$ then $r_{1}>0$. Now we shall show that $B_{e}\left(z, r_{1}\right) \subset B_{e}\left(x_{0}, r\right)$. Suppose $u \in$ $B_{e}\left(z, r_{1}\right)$. Then we have $\|z-u, u-e, e-z\|<r_{1}$. Now

$$
\begin{aligned}
&\left\|x_{0}-e, e-u, u-x_{0}\right\|=\left\|x_{0}-z+z-e, e-u, z-x_{0}+u-z\right\| \\
& \leq\left\|x_{0}-z, 0, z-x_{0}\right\|+\|z-e, e-u, u-z\| \\
&<\left\|x_{0}-z, 0, z-x_{0}\right\|+r_{1}=r \\
& \Rightarrow\left\|x_{0}-e, e-u, u-x_{0}\right\|<r
\end{aligned}
$$

Therefore $u \in B_{e}\left(x_{0}, r\right)$. Hence the result.

Proposition 2.6 For $a \in X$ and $r>0$, We have $\overline{B_{e}(a, r)} \subseteq B_{e}[a, r]$.

Proof. We prove the result by showing thaty $\notin B_{e}[a, r] \Longrightarrow y \notin \overline{B_{e}(a, r)}$.

If $r_{1}=\|y-a, a-e, e-y\|$, then $r_{1}>r$. 
Let $x \in B_{e}(a, r)$. Then $\|x-a, a-e, e-x\|<r$. Now

$$
\begin{aligned}
\|y-a, a-e, e-y\| & =\|y-x+x-a, a-e, x-y+e-x\| \\
& \leq\|y-x, 0, x-y\|+\|x-a, a-e, e-x\| \\
\Rightarrow\|y-x, 0, x-y\| & \geq\|y-a, a-e, e-y\|-\|x-a, a-e, e-x\| \\
& >r_{1}-r>0
\end{aligned}
$$

Let $\varepsilon=r_{1}-r$. Then $x \notin B_{y}(y, \varepsilon)$. Hence there exists a neighborhood of $y$ which does not intersect $B_{e}(a, r)$, i.e. $y \notin \overline{B_{e}(a, r)}$. Hence the result.

Proposition 2.7 The balls $B_{0}(0, r)$ and $B_{0}[0, r]$ are absolutely convex for every $r>0$.

Proof. Let $x, y \in B_{0}(0, r)$. Then $\|x,-x, 0\|<r,\|y,-y, 0\|<r$. Let $\alpha, \beta \in \mathbb{R}$ with $|\alpha|+|\beta| \leq 1$. Then

$$
\begin{aligned}
\|\alpha x+\beta y,-\alpha x-\beta y, 0\| & \leq\|\alpha x,-\alpha x, 0\|+\|\beta y,-\beta y, 0\| \\
& =|\alpha|\|x,-x, 0\|+|\beta|\|y,-y, 0\| \\
& <(|\alpha|+|\beta|) r \\
& \leq r
\end{aligned}
$$

This implies that $\alpha x+\beta y \in B_{0}(0, r)$. Hence $B_{0}(0, r)$ is absolutely convex. Similarly, we can show that the ball $B_{0}[0, r]$ is absolutely convex.

Proposition 2.8 The closure of a convex (resp. absolutely convex) subset of a G-normed space is convex (resp. absolutely convex).

Proof. Let $X$ be a G-normed space. Let $A$ be a convex (resp. absolutely convex) subset of $X$. Let $x, y \in \bar{A}$. Then there exist sequences $\left\langle x_{n}\right\rangle$ and $\left\langle y_{n}>\right.$ in $A$ such that $x_{n} \rightarrow x$ and $y_{n} \rightarrow y$.

Let $\alpha, \beta>0(\operatorname{resp} . \alpha, \beta \in \mathbb{R})$ such that $\alpha+\beta=1(\operatorname{resp} .|\alpha|+|\beta| \leq 1)$. Since $A$ is convex (resp. absolutely convex), $\alpha x_{n}+\beta y_{n} \in A$ for all $n \in \mathbb{N}$. Now

$$
\alpha x+\beta y=\lim _{n \rightarrow \infty}\left(\alpha x_{n}+\beta y_{n}\right) \in \bar{A}
$$

Hence $A$ is convex (resp. absolutely convex).

Theorem 2.1 Let $X$ be a G-normed space. Then the following maps are continuous:

(a) Addition: $X \times X \rightarrow X, \quad(x, y) \mapsto x+y$,

(b) Scalar multiplication: $\mathbb{R} \times X \rightarrow X,(\lambda, x) \mapsto \lambda x$,

(c) The G-norm: $X \times X \times X \rightarrow \mathbb{R},(x, y, z) \mapsto\|x, y, z\|$ 
Proof. We may regard $X$ and $\mathbb{R}$ as metric spaces. Let $\left\langle x_{n}\right\rangle,\left\langle y_{n}\right\rangle$ and $\left\langle z_{n}\right\rangle$ be sequences in a Gnormed space $X$ with $\lim _{n \rightarrow \infty} x_{n}=x, \lim _{n \rightarrow \infty} y_{n}=y$ and $\lim _{n \rightarrow \infty} z_{n}=z$. Let $\left\langle a_{n}>\right.$ be a sequence in $\mathbb{R}$ with $\lim _{n \rightarrow \infty} a_{n}=a$

(a) We have

$$
\begin{array}{r}
\begin{aligned}
\left\|x_{l}+y_{l}-(x+y), x_{m}+y_{m}-(x+y), x_{n}+y_{n}-(x+y)\right\| & \left.\leq \| x_{l}-x, x_{m}-x, x_{n}-x\right) \| \\
& +\left\|y_{l}-y, y_{m}-y, y_{n}-y\right\|
\end{aligned} \\
\text { Hence } \lim _{m, n \rightarrow \infty}\left\|x_{l}+y_{l}-(x+y), x_{m}+y_{m}-(x+y), x_{n}+y_{n}-(x+y)\right\|=0
\end{array}
$$

This proves the result.

(b) The result follows by similar arguments.

(c) Using the relation (2.1), we have

$$
\left|\left\|x_{n}, y_{n}, z_{n}\right\|-\|x, y, z\|\right| \leq\left\|x_{n}-x, y_{n}-y, z_{n}-z\right\|
$$

Therefore $\lim _{n \rightarrow \infty}\left\|x_{n}, y_{n}, z_{n}\right\|=\|x, y, z\|$. Hence the result.

The following result holds for any topological vector space, hence we state it without proof.

Proposition 2.9 The intersection of a finite number of dense open subsets of a G-normed space $X$ is dense in $X$.

Definition 2.11 A linear function $F$ from a G-normed space $\left(X,\|., \ldots\|_{X}\right)$ into a G-normed space $\left(Y,\|., \ldots,\|_{Y}\right)$ is said to be bounded if there exists $K>0$ such that

$$
\|F(x), F(y), F(z)\|_{Y} \leq K\|x, y, z\|_{X} \quad \forall x, y, z \in X
$$

Definition 2.12 A linear function $F$ from a G-normed space $\left(X,\|., \ldots\|_{X}\right)$ into a G-normed space $\left(Y,\|., \ldots,\|_{Y}\right)$ is continuous at $x_{0} \in X$ if there exists a sequence $<x_{n}>$ in $X$ such that

$$
x_{n} \rightarrow x_{0} \Rightarrow F\left(x_{n}\right) \rightarrow F\left(x_{0}\right)
$$

Equivalently, $F$ is continuous at a point $x_{0}$ if for given $z \in X$ and $\varepsilon>0$ there exists $\delta>0$ and $u \in X$ such that $\left\|F(y)-F\left(x_{0}\right), F\left(x_{0}\right)-F(z), F(z)-F(y)\right\|_{Y}<\varepsilon$ for every $y \in X$ for which $\| y-x_{0}, x_{0}-$ $u, u-y \|_{X}<\delta$.

$F$ is continuous if it is continuous at every point in $X$.

From now on we denote $\|., \ldots,\|_{X}\left(\right.$ or $\left.\|., \ldots,\|_{Y}\right)$ simply by $\|., \ldots$,$\| .$

Theorem 2.2 Every bounded linear function is continuous.

Proof. Let $F$ be a bounded linear function from a G-normed space $(X,\|., \ldots\|$.$) into a G-normed$ space $(Y,\|., .,\|$.$) . Then there exists K>0$ such that

$$
\|F(u), F(v), F(w)\| \leq K\|u, v, w\| \quad \forall u, v, w \in X
$$


Hence for $x, y, z \in X$ and given $\varepsilon>0$ there exists $\delta=\varepsilon / K>0$ such that $\| F(y-x), F(x-z), F(z-$ $x) \|<\varepsilon$ whenever $\|y-x, x-z, z-x\|<\delta$. Since $F$ is linear, hence $\| F(y)-F(x), F(x)-$ $F(z), F(z)-F(x) \|<\varepsilon$ whenever $\|y-x, x-z, z-x\|<\delta$, i.e. $F$ is continuous.

We now state and prove the famous Banach's fixed point theorem for G-normed spaces.

Theorem 2.3 Let $X$ be a G-Banach space and $T: X \rightarrow X$ be a mapping satisfying the following condition for all $x, y, z \in X$

$$
\|T x-T y, T y-T z, T z-T x\| \leq k\|x-y, y-z, z-x\|
$$

Where $k \in[0,1)$. Then $T$ has a unique fixed point.

Proof. Let $T: X \rightarrow X$ be a mapping satisfying the condition (2.6). Let $x_{0} \in X$ be an arbitrary point. Define a sequence $<x_{n}>$ by the relation $x_{n}=T^{n} x_{0}$, then by the given condition we have

$$
\left\|T x_{n-1}-T x_{n}, T x_{n}-T x_{n-1}, 0\right\| \leq k\left\|x_{n-1}-x_{n}, x_{n}-x_{n-1}, 0\right\|
$$

Or

$$
\left\|x_{n}-x_{n+1}, x_{n+1}-x_{n}, 0\right\| \leq k\left\|x_{n-1}-x_{n}, x_{n}-x_{n-1}, 0\right\|
$$

Continuing the same argument, we have

$$
\left\|x_{n}-x_{n+1}, x_{n+1}-x_{n}, 0\right\| \leq k^{n}\left\|x_{0}-x_{1}, x_{1}-x_{0}, 0\right\|
$$

For all natural numbers $n$ and $m(>n)$, by using [N 4] we have

$$
\begin{gathered}
\left\|x_{n}-x_{m}, x_{m}-x_{n}, 0\right\| \leq\left\|x_{n}-x_{n+1}, x_{n+1}-x_{n}, 0\right\|+\left\|x_{n+1}-x_{n+2}, x_{n+2}-x_{n+1}, 0\right\| \\
+\cdots+\left\|x_{m-1}-x_{m}, x_{m}-x_{m-1}, 0\right\|
\end{gathered}
$$

Since $0 \leq k<1$, hence on using the relation (2.7), we get

$$
\begin{aligned}
\left\|x_{n}-x_{m}, x_{m}-x_{n}, 0\right\| & \leq\left(k^{n}+k^{n+1}+\cdots+k^{m-1}\right)\left\|x_{0}-x_{1}, x_{1}-x_{0}, 0\right\| \\
& \leq \frac{k^{n}}{1-k}\left\|x_{0}-x_{1}, x_{1}-x_{0}, 0\right\|
\end{aligned}
$$

This yields $\left\|x_{n}-x_{m}, x_{m}-x_{n}, 0\right\| \rightarrow 0$ as $m, n \rightarrow \infty$. Now

$$
\begin{aligned}
\left\|x_{l}-x_{m}, x_{m}-x_{n}, x_{n}-x_{l}\right\| & =\left\|x_{l}-x_{m}, x_{m}-x_{l}+x_{l}-x_{n}, x_{n}-x_{l}\right\| \\
& \leq\left\|x_{l}-x_{m}, x_{m}-x_{l}, 0\right\|+\left\|0, x_{l}-x_{n}, x_{n}-x_{l}\right\|
\end{aligned}
$$

Therefore $\lim _{l, m, n \rightarrow \infty}\left\|x_{l}-x_{m}, x_{m}-x_{n}, x_{n}-x_{l}\right\|=0$ and hence $\left\langle x_{n}>\right.$ is a Cauchy sequence. Since $X$ is complete, there exists $u \in X$ such that $x_{n} \rightarrow u$.

Suppose that $T u \neq u$, then

$$
\begin{aligned}
\|T u-u, u-T u, 0\| & \leq\left\|T u-T x_{n}, T x_{n}-T u, T u-T u\right\|+\left\|T x_{n}-u, u-T x_{n}, 0\right\| \\
& \leq k\left\|u-x_{n}, x_{n}-u, 0\right\|+\left\|x_{n+1}-u, u-x_{n+1}, 0\right\|
\end{aligned}
$$


Taking the limits as $n \rightarrow \infty$ and using the fact that the G-norm is a continuous function of its variables, we observe that LHS is independent of $n$ and RHS tends to zero. Hence we must have Tu=u.

For uniqueness of $u$, suppose that $v \neq u$ is such that $T v=v$. Then we have

$$
\|v-u, 0, u-v\|=\|T v-T u, 0, T u-T v\| \leq k\|v-u, 0, v-u\|
$$

Which yields a contradiction as $0 \leq k<1$. Hence we have $u=v$.

Definition 2.13 Let $X$ be a $G$-normed space and $T$ be a self mapping on $X$. Then $T$ is called expansive mapping if there exists a constant $q>1$ such that for all $x, y, z \in X$, we have

$$
\|T x-T y, T y-T z, T z-T x\| \geq q\|x-y, y-z, z-x\|
$$

Theorem 2.4 Let $T$ be a linear surjective self mapping on a $G$-Banach space $X$ satisfying the condition (2.8). Then $T$ has a unique fixed point.

Proof. First we see that $T$ is invertible, for if $T x=T y$, taking $x, y, y$ for $x, y, z$, condition (2.8) gives $x=$ $y$, i.e. $T$ is injective and hence invertible.

Let $S$ be the inverse mapping of $T$. Then $S$ is linear and

$$
\begin{gathered}
\|x-y, y-z, z-x\|=\|T S(x-y), T S(y-z), T S(z-x)\| \geq q\|S x-S y, S y-S z, S z-S x\| \\
\|S x-S y, S y-S z, S z-S x\| \leq k\|x-y, y-z, z-x\| \text { where } k=1 / q .
\end{gathered}
$$

or

Hence by Theorem 2.3 the mapping $S$ has a unique fixed point $u \in X$ such that $S u=u$. Now $u=$ $(T S) u=T(S u)=T u$. Thus $u$ is also a fixed point of $T$.

If there exists some $v \neq u$ such that $T v=v$, then $T v=v=(T S) v=(S T) v=S(T v)$, i.e. $T v$ is another fixed point of $S$. By uniqueness of fixed point for $S$ we conclude that $u=T v=v$, i.e. $u$ is a fixed point of $T$.

Theorem 2.5 Let $X$ be a $G$-Banach space and let $T$ and $S$ be self mappings on $X$ satisfying the following conditions:

(1) $T(X) \subseteq S(X)$

(2) $S$ is continuous,

(3) $\|T x-T y, T y-T z, T z-T x\| \leq q\|S x-S y, S y-S z, S z-S x\|$ for every $\quad x, y, z \in X$ and $0<q<1$.

Then $T$ and $S$ have a unique common fixed in $X$ provided $T$ and $S$ commute.

Proof. Let $x_{0}$ be an arbitrary point in $X$. Since $T(X) \subseteq S(X)$ hence there exists a point $x_{1}$ such that $T x_{0}=$ $S x_{1}$. In general we can choose $x_{n+1}$ such that $y_{n}=T x_{n}=S x_{n+1}$. From (3) we have

$$
\begin{aligned}
\left\|T x_{n}-T x_{n+1}, T x_{n+1}-T x_{n}, T x_{n}-T x_{n}\right\| & \leq q\left\|S x_{n}-S x_{n+1}, S x_{n+1}-S x_{n}, S x_{n}-S x_{n}\right\| \\
& =q\left\|T x_{n-1}-T x_{n}, T x_{n}-T x_{n-1}, 0\right\|
\end{aligned}
$$


Proceeding in above manner we have

$$
\begin{gathered}
\left\|T x_{n}-T x_{n+1}, T x_{n+1}-T x_{n}, T x_{n}-T x_{n}\right\| \leq q^{n}\left\|T x_{0}-T x_{1}, T x_{1}-T x_{0}, 0\right\| \\
\left\|y_{n}-y_{n+1}, y_{n+1}-y_{n}, 0\right\| \leq q^{n}\left\|y_{0}-y_{1}, y_{1}-y_{0}, 0\right\|
\end{gathered}
$$

Hence for all natural numbers $n$ and $m(>n)$, it can be shown that

$$
\left\|y_{n}-y_{m}, y_{m}-y_{n}, 0\right\| \leq \frac{q^{n}}{1-q}\left\|y_{0}-y_{1}, y_{1}-y_{0}, 0\right\|
$$

This yields $\left\|y_{n}-y_{m}, y_{m}-y_{n}, 0\right\|$ as $m, n \rightarrow \infty$. Now

$$
\left\|y_{l}-y_{m}, y_{m}-y_{n}, y_{n}-y_{l}\right\| \leq\left\|y_{l}-y_{m}, y_{m}-y_{l}, 0\right\|+\left\|0, y_{l}-y_{n}, y_{n}-y_{l}\right\| \rightarrow 0
$$

as $l, m, n \rightarrow \infty$. Hence $\left\langle y_{n}>\right.$ is a Cauchy sequence. Since $X$ is complete, there exists $u \in X$ such that $y_{n} \rightarrow u$. Since $y_{n}=T x_{n}=S x_{n+1}$, hence we have $\lim _{n \rightarrow \infty} y_{n}=\lim _{n \rightarrow \infty} T x_{n}=\lim _{n \rightarrow \infty} S x_{n}=u$. Now $S$ is continuous hence

$$
\lim _{n \rightarrow \infty} S S x_{n}=\lim _{n \rightarrow \infty} S T x_{n}=S u
$$

Also $T$ and $S$ commute, therefore

$$
\lim _{n \rightarrow \infty} T S x_{n}=\lim _{n \rightarrow \infty} S T x_{n}=\lim _{n \rightarrow \infty} S S x_{n}=S u
$$

Taking $x=S x_{n}, y=x_{n}$ and $z=x_{n}$ in (3) we have

$$
\left\|T S x_{n}-T x_{n}, 0, T x_{n}-T S x_{n}\right\| \leq q\left\|S S x_{n}-S x_{n}, 0, S x_{n}-S S x_{n}\right\|
$$

Making $n \rightarrow \infty$, we have $\|S u-u, 0, u-S u\| \leq q\|S u-u, 0, u-S u\|$. Which gives $S u=u$. For otherwise $q \geq 1$ contradicting the fact that $0<q<1$.

Similarly on taking $x=x_{n}, y=u$ and $z=u$ in (3) and making $n \rightarrow \infty$, we have $T u=u$. Therefore $T u=S u=u$, i.e. $u$ is a common fixed point of $T$ and $S$.

For uniqueness of $u$, suppose that $v \neq u$ is such that $T v=S v=v$. Then we have

$$
\|u-v, 0, v-u\|=\|T u-T v, 0, T v-T u\| \leq q\|S u-S v, 0, S v-S u\|<\|u-v, 0, v-u\|
$$

Thus we get a contradiction, hence we have $u=v$.

\section{References}

[1] B.C. Dhage, A study of some fixed point theorem, Ph.D. Thesis, Marathwada Univ. Aurangabad, 1984.

[2] R. Freese, Y.J. Cho, and S. S. Kim, Strictly 2-convex linear 2-normed spaces, J. Korean Math. Soc. 29 (1992), 391-400.

[3] R. Freese and Y.J. Cho, Geometry of Linear 2-normed Spaces, Nova Science Publ., New York, 2001. 
[4] S. Gähler, 2-metrische Räume und ihre topologische struktur, Math. Nachr. 26 (1963), 115-148.

[5] S. Gähler, Lineare 2-normierte Räume, Math. Nachr. 28 (1964), 1-43.

[6] S. Gähler, Untersuchungen über verallgemeinerte m-metrische Räume. I, Math. Nachr. 40 (1969), 165-189.

[7] S. Gähler, Untersuchungen über verallgemeinerte m-metrische Räume. II, Math. Nachr. .40 (1969), 229-264.

[8] S. Gähler, Untersuchungen über verallgemeinerte m-metrische Räume. III, Math. Nachr. .41 (1969), 23-36.

[9] H. Gunawan, and Mashadi, On n-normed spaces, Int. J. Math. Math. Sci. 27 (2001) 631-639.

[10] K.S. Ha, Y.J. Cho and A. White, Strictly convex and 2-convex 2-normed spaces, Math. Japonica 33 (1988), 375-384.

[11] K.A. Khan, Generalized $n$-metric spaces and fixed point theorems, to appear in Journal of Nonlinear and Convex Analysis.

[12] A. Misiak, $n$-inner product spaces, Math.Nachr. 140 (1989), 299-319.

[13] Z. Mustafa, and B. Sims, Some remarks concerning D-metric spaces, Proceedings of the International Conferences on Fixed Point Theory and Applications, Valencia, Spain, July (2003), 189198.

[14] Z. Mustafa, and B. Sims, A new approach to generalized metric spaces, Journal of Nonlinear and Convex Analysis 7 (2006), 289-297.

[15] A.K. Sharma, A note on fixed points in 2-metric spaces, Indian J. Pure Appl. Math. 11 (1980), 15801583.

[16] R. K. Vats, S. Kumar, and V. Sihag, Common fixed point theorem for expansive mappings in Gmetric spaces, Journal of Mathematics and Computer Science 6 (2013), 60-71. 